\title{
Diferencias anatómicas associades a la sensibilidad al castigo y a la recompensa: un estudio de morfometría basada en el vóxel
}

\author{
JESÚS ADRIÁN-VENTURA \\ jadrian@uji.es \\ VÍctor COSTUMERo \\ vcostume@uji.es \\ ALFONSO BARRÓS-LOSCERTALES \\ barros@uji.es \\ CÉSAR ÁVILA \\ avila@uji.es
}

\section{Resumen}

Introducción: La teoría de la sensibilidad al refuerzo (TSR) propone el sistema de activación conductual (SAC) como el sistema motivacional encargado de dirigir la conducta hacia la obtención de estímulos apetitivos. A nivel neurobiológico, este sistema se ha relacionado con el circuito cerebral de la recompensa. Así mismo, se ha propuesto el rasgo de personalidad de sensibilidad a la recompensa (SR) como modulador de la actividad del SAC. En el presente trabajo estudiamos la relación entre la actividad del SAC, medida por medio del cuestionario SPSRQ, y el volumen de sustancia gris en áreas de recompensa. Métodos: Realizamos un análisis de morfometría basada en el vóxel (VBM) en una muestra de 216 hombres e investigamos la asociación entre los valores de volumen obtenidos y las puntuaciones en la escala SR del SPSRQ mediante un análisis de regresión. El procesado y análisis de las imágenes se realizó mediante SPM8. Resultados: A nivel cortical, los análisis de VBM mostraron una correlación negativa entre las puntuaciones de la escala SR y el volumen de sustancia gris en el córtex prefrontal lateral, la ínsula y el lóbulo temporal superior. A nivel subcortical, observamos una reducción en el volumen del estriado (núcleo caudado). Conclusiones: Nuestros resultados apoyan investigaciones previas y aportan nuevas evidencias acerca de las diferencias anatómicas en áreas relacionadas con el control inhibitorio y la toma de decisiones en participantes con un SAC sobreactivado.

Palabras clave: recompensa, morfometría, córtex prefrontal, estriado, modelo de Gray.

\section{Abstract}

Introduction: The Reinforcement Sensitivity Theory (RST) proposes the Behavioral Activation System (BAS) as the motivational system responsible for direct behavior to- 
ward appetitive stimuli. Neurobiologically, this system has been related to the brain reward circuitry. Additionally, the personality trait of Sensitivity to Reward (SR) has been proposed as modulator of BAS activity. In this work, we studied the relationship between the BAS activity, as measured by the SPSRQ questionnaire, and the gray matter volume in reward-related areas. Methods: We carried out a voxel-based morphometry analysis in a sample of 216 male participants and we investigated the association between the data volume obtained and the SR scale scores by means of a regression analysis. Image processing and statistical analyses were carried out using SPM8. Results: At the cortical level, VBM analysis showed a negative correlation between SR scores and the gray matter volume in the lateral prefrontal cortex, the insula, and the superior temporal lobe. Subcortically, we found a reduction in the striatum volume (caudate nucleus). Conclusion: Our results support previous studies and provide new evidence about anatomical differences in brain areas related to inhibitory control and decision-making in participants with an overactive BAS.

Keywords: reward, morphometry, prefrontal cortex, striatum, Gray's model.

\section{Introducción}

La teoría de la sensibilidad al refuerzo (TSR) propuesta por Gray (1982), y más tarde revisada por Gray y McNaughton (2000), propone el SAC como el sistema cerebral encargado de responder a estímulos condicionados e incondicionados apetitivos. Cuando uno de estos estímulos está presente, se dan dos efectos mediados por el SAC: un efecto motivacional, debido a un incremento en el arousal (Barrós-Loscertales y cols., 2010; Corr, 2004), que estimula y promueve la conducta de aproximación hacia la obtención de la recompensa, y un efecto en el aprendizaje, debido a que se redirige la atención sobre el estímulo de recompensa, facilitando el aprendizaje de relaciones estímulo-estímulo y estímulo-respuesta (Pickering y Gray, 2001). De esta forma, en base al aprendizaje de contingencias reforzantes, se ha relacionado el SAC con el afecto positivo y las expectativas de recompensa (Cohen, 2007; Schultz, 2000). Como resultado, se ha podido comprobar que el SAC facilita la detección de estímulos condicionados asociados a recompensas y promueve conductas de aproximación hacia estos (Corr, 2004).

En relación a las bases biológicas, el sistema dopaminérgico ha sido propuesto como elemento central en el funcionamiento del SAC, a causa de la implicación de las vías mesolímbicas y mesocorticales en la conducta dirigida a la obtención de recompensas (Depue y Collins, 1999; Pickering y Gray, 2001). Dentro de este sistema se incluyen áreas corticales, como el córtex prefrontal medial, el córtex orbitofrontal, la corteza cingulada anterior y la ínsula. A nivel subcortical encontramos el área tegmental ventral, la sustancia negra, los ganglios basales y la amígdala (Haber y Knutson, 2010; Sescousse, Caldú, Segura y Dreher, 2013).

La TSR propone la existencia de diferencias individuales en la reactividad del SAC (Gray, 1982; Gray y McNaughton, 2000). De esta forma, en la TSR se relacionan factores motivacionales y emocionales que estarían asociados con la disposición de respuesta y que, a su vez, están modulados por la actividad del SAC (Corr, 2004). Así mismo, la actividad del SAC se ha asociado al rasgo de personalidad de sensibilidad a la recompensa (SR). En relación a esto, algunos estudios han relacionado la sobreactivación del SAC con la sensibilización del sistema dopaminérgico mesencefálico (Robinson y Berridge, 2008; Vezina, 2004), lo cual también está 
relacionado con una mayor SR. Una alta SR provoca que los individuos detecten más estímulos apetitivos y, una vez detectados, que tengan una mayor probabilidad de llevar a cabo la conducta de aproximación (Corr, 2004; Gray y McNaughton, 2000). Así pues, la relación entre el SAC y la SR también puede ser de utilidad a la hora de evaluar rasgos de impulsividad (Torrubia, Ávila, Moltó y Caseras, 2001).

La investigación en personalidad ha propuesto la existencia de diferencias individuales asociadas a la actividad del SAC. Estas diferencias, asociadas a la SR, pueden ser evaluadas mediante medidas de autoinforme, como por ejemplo los cuestionarios de sensibilidad al castigo y sensibilidad a la recompensa (SPSRQ; Torrubia y cols., 2001) y la escala BIS/BAS (Carver y White, 1994). En base a esto, en los últimos años, diversos estudios de neuroimagen han analizado las diferencias anatómicas y funcionales asociadas a la actividad del SAC. Así, se ha podido comprobar que las personas con un SAC sobreactivado muestran una reducción en el volumen de sustancia gris en áreas frontoestriatales (Barrós-Loscertales y cols., 2006), lo cual también se ha visto en relación a la impulsividad (Muhlert y Lawrence, 2015). Estas diferencias también se han asociado a trastornos del control de impulsos, como el abuso de sustancias, donde también se han observado reducciones en el lóbulo temporal y la ínsula (Barrós-Loscertales y cols., 2011; Moreno-López y cols., 2012).

En referencia a las diferencias funcionales, estudios de resonancia magnética funcional (RMf) han evidenciado la modulación que ejerce el SAC en áreas cerebrales involucradas en el circuito de la recompensa (Barrós-Loscertales y cols., 2010; Costumero y cols., 2013). En estos estudios se observó una hiperactivación en áreas relacionadas con la detección y el procesamiento de recompensas, como la amígdala, la ínsula, el estriado dorsal y ventral y áreas prefrontales ante la presentación de estímulos recompensantes (véase Kennis y cols., 2013 para revisión).

Por otra parte, la reactividad del sistema de recompensa también se ha asociado a trastornos clínicos. Una alta SR se ha relacionado con trastornos clínicos caracterizados por la falta de control de impulsos, como por ejemplo el abuso de sustancias (Balconi, Finocchiaro y Campanella, 2014; Franken, Muris y Georgieva, 2006), el trastorno por déficit de atención (TDAH) con hiperactividad (Mitchell y Nelson-Gray, 2006) o la obesidad (Shott y cols., 2014). Por el contrario, una baja SR se ha relacionado con trastornos caracterizados por la falta de motivación hacia estímulos apetitivos, como por ejemplo la depresión (Kimbrel, Nelson-Gray y Mitchell, 2007; Pinto-Meza y cols., 2006).

Pese a la aceptación del modelo de Gray, en la actualidad no hay una gran evidencia acerca de las diferencias cerebrales asociadas a la actividad del SAC. Por ello, el objetivo de nuestro estudio fue analizar la relación entre las diferencias individuales en la actividad de este sistema, medida por medio de la escala SR del cuestionario SPSRQ, y el volumen de sustancia gris en áreas relacionas con el circuito de la recompensa en una amplia muestra. Decidimos realizar nuestro estudio únicamente en hombres debido a que estudios previos han evidenciado diferencias de género tanto en volumen cerebral (Good y cols., 2001) como en las puntaciones en SR (Torrubia y cols., 2001).

\section{Métodos}

\section{Participantes}

Para este estudio seleccionamos una muestra formada por 216 hombres, con una media de edad de 23,46 años (D. E. = 4,55; rango = 18-39 años). El requisito que debían cumplir para poder participar en el estudio fue no tener antecedentes de enfermedad neurológica o psiquiátrica y no haber tenido ningún traumatismo con pérdida de conciencia. Además, los 
participantes fueron informados de la naturaleza del estudio y firmaron el consentimiento por escrito. El estudio fue aprobado por el Comité de Ética de la Universitat Jaume I.

Todos los participantes completaron el cuestionario de sensibilidad al castigo y a la recompensa (ScSR; Torrubia y cols., 2001), obteniendo una puntuación media para la escala de SR de 11,93 (D. E. = 4,80; rango = 3-23).

\section{Adquisición de datos de resonancia magnética}

Las imágenes se obtuvieron por medio de un escáner Siemens Avanto (Erlangen, Alemania) de 1.5 T. Los participantes fueron colocados en posición supina en el escáner de resonancia magnética y se les inmovilizó la cabeza mediante cojines para reducir los artefactos producidos por el ruido.

Para la adquisión de los datos estructurales, se adquirió un volumen 3D potenciado en T1 usando una secuencia MPRAGE (TE $=3,8 \mathrm{~ms}$; TR $=2200 \mathrm{~ms}$; FOV $=256 \mathrm{~mm}$; matriz $=256 \times 256 \times 160$; tamaño del voxel $=1 \times 1 \times 1 \mathrm{~mm})$.

\section{Morfometría basada en el vóxel}

El análisis de morfometría basada en el vóxel (VBM) se realizó mediante la herramienta VBM8, implementada para el el paquete estadístico SPM8 (Statistical Parametric Mapping, WeIlcome Institute of Cognitive Neurology, Londres, RU). El preprocesado de las imágenes originales de todos los participantes se realizó en base al proceso automatizado de segmentación unificada (Ashburner y Friston, 2005). Las imágenes fueron segmentadas en sustancia gris, sustancia blanca y líquido cefalorraquídeo y fueron normalizadas a un espacio estereotáxico estándar (plantilla proporcionada por el International Consortium of Brain Mapping, ICBM) mediante transformaciones lineales (12 parámetros) y no lineales. Para lograr un registro espacial más preciso, creamos una plantilla específica para nuestro estudio, para posteriormente realizar una normalización mediante DARTEL. Los parámetros resultantes de la normalización fueron aplicados a las imágenes originales, siendo moduladas con una interpolación trilineal a un tamaño final de vóxel de $1 \mathrm{~mm}^{3}$. Los determinantes Jacobianos derivados de la normalización espacial fueron aplicados a los valores de vóxel originales, permitiendo que las estructuras conservaran la informacion de su volumen previo. Finalmente, las imágenes fueron suavizadas con un filtro gaussiano de $10 \mathrm{~mm}$.

\section{Análisis estadístico}

El análisis de regresión vóxel a vóxel fue realizado tomando el volumen de sustancia gris como variable dependiente y las puntuaciones en la escala SR como covariable de interés, dentro del marco del modelo lineal general en SPM8. La variable edad tambén fue incluida como covariable, a fin de controlar posibles efectos en el volumen cerebral.

El umbral estadístico establecido para el análisis de todo el cerebro (whole-brain) fue de $p<0.05$ corregido por comparaciones múltiples (FWE corregido a nivel de cluster). Para el análisis de regiones de interés (ROI), establecimos un umbral de $p<0.05$ corregido por comparaciones múltiples (FWE corregido a nivel de vóxel).

Las ROI analizadas fueron definidas por medio del Wake Forest University PickAtlas (Maldjian, Laurienti, Kraft y Burdette, 2003) 
. Así mismo, las ROI para el córtex orbitofrontal, la amígdala, el cingulado anterior y el núcleo caudado fueron extraídas por medio del atlas AAL (automated anatomical labeling). Para el núcleo accumbens, definimos dos esferas de $6 \mathrm{~mm}$ usando las coordenadas MNI ( $x y, z= \pm 12,8,-4)$ provistas en el metaanálisis de Liu, Hairston, Schrier y Fan (2011).

\section{Resultados}

En relación a la actividad del SAC, el análisis para todo el cerebro nos permitió detectar cinco clusters de sustancia gris que mostraron una correlación negativa con las puntuaciones en la escala SR. Estos fueron localizados en el córtex prefrontal lateral, el lóbulo temporal superior, la ínsula, el córtex cingulado medio y el giro occipital (véase la tabla 1, figura 1). No se encontraron correlaciones positivas significativas entre las puntuaciones de SR y el volumen de sustancia gris.

Tabla 1

Áreas cerebrales que muestran una correlación negativa entre el volumen de sustancia gris y las puntuaciones en la escala $S R$

\begin{tabular}{lcccc}
\hline \multicolumn{1}{c}{ Área } & Hemisferio & $\begin{array}{c}\text { Coordenadas } \\
\text { MNI x y, z }\end{array}$ & T & k-vóxels \\
\hline Frontal inferior opercular & I & $-60,11,21$ & 4,38 & 4324 \\
Temporal superior & I & $-47,-7,-9$ & 4,34 & \\
Giro precentral & I & $-54,2,28$ & 4,33 & \\
Opérculo rolándico & I & $-59,0,6$ & 3,65 & 3048 \\
Ínsula & I & $-47,3,-0$ & 3,44 & \\
Frontal medio & I & $-30,56,22$ & 4,68 & 1697 \\
Giro frontal superior & I & $-32,50,37$ & 4,28 & \\
Frontal superior & I & $-26,60,6$ & 4,25 & \\
Frontal superior & D & $24,62,12$ & 3,78 & 1528 \\
Frontal superior medial & D & $12,60,16$ & 3,70 & \\
Frontal superior medial & I & $-6,47,51$ & 3,43 & \\
Cingulado medio & I & $-9,-28,33$ & 4,82 & \\
Cingulado medio & D & $6,-19,34$ & 4,22 & \\
Giro occipital medio & I & $-51,-79,6$ & 4,12 & 4,09 \\
Giro temporal inferior & I & $-56,-73,-6$ & 4,02 & \\
Temporal medio & $-54,-61,21$ & & \\
\hline
\end{tabular}

D: derecho; I: izquierdo; $p<0,05$ FWE corregido 


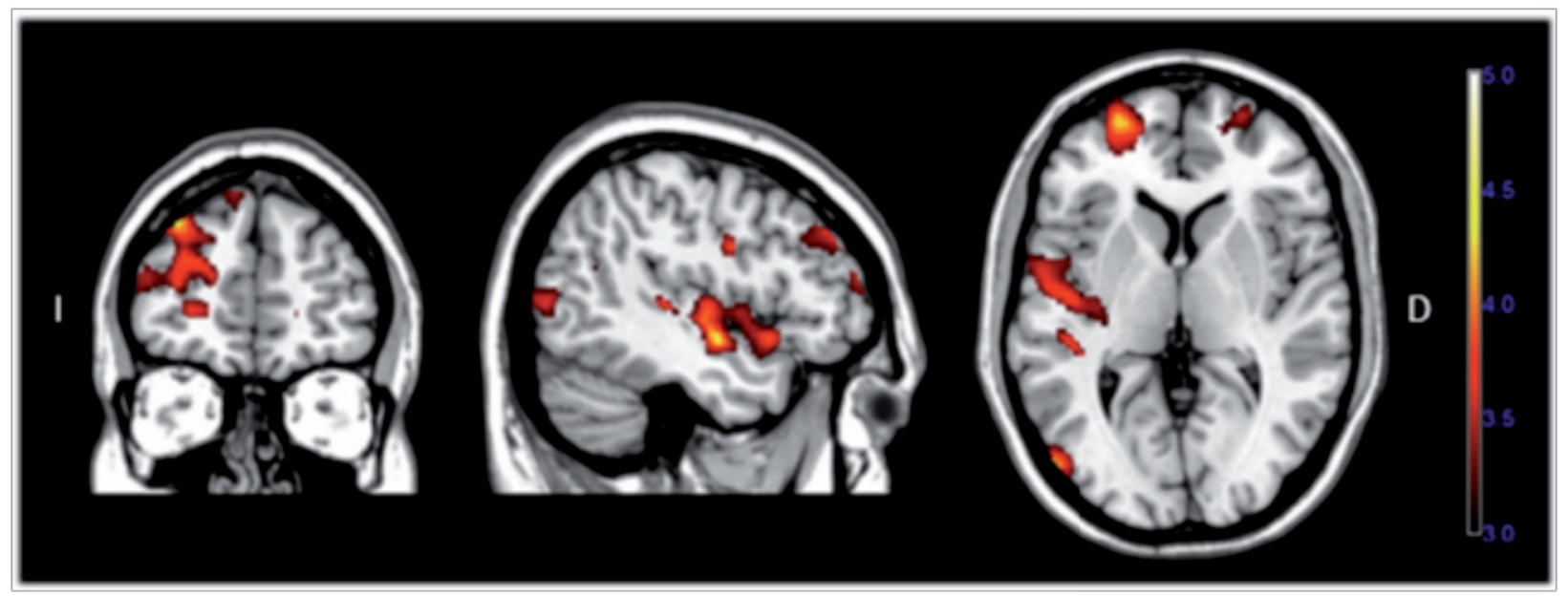

Figura 1. Áreas cerebrales relacionadas con el procesamiento de recompensas (córtex prefrontal bilateral, ínsula izquierda y lóbulo temporal superior izquierdo) que muestran una correlación negativa con las puntuaciones de la escala SR ( $p<0,05$, corregido FWE). La barra de color muestra los valores T asociados. D: derecha; I: izquierda

Por su parte, el análisis de regresión en las RO mostró únicamente una correlación negativa entre el volumen de sustancia gris en el estriado izquierdo (máxima local en $x y, z=-11,21,-2$; $\mathrm{T}=2,96 ; \mathrm{k}=117 ; \mathrm{p}<0,05$, FWE corregido) y las puntuaciones en la escala SR (figura 2).

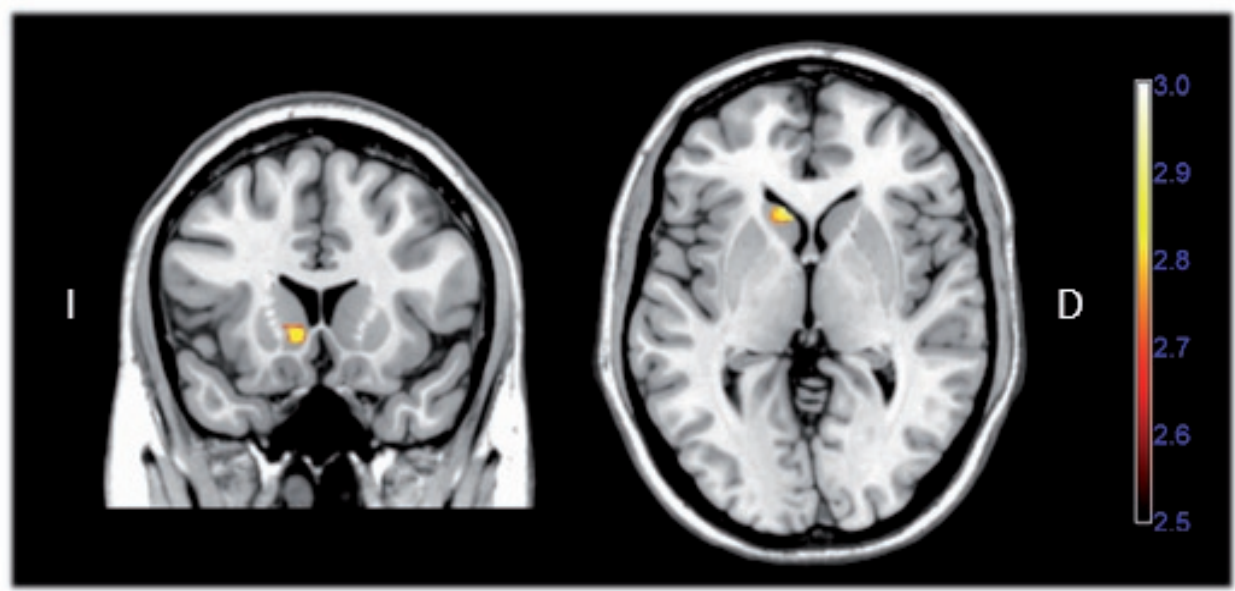

Figura 2. Correlación negativa entre el volumen de sustancia gris en el estriado ventral izquierdo (parte ventral del núcleo caudado) y las puntuaciones en la escala $\mathrm{SR}$ ( $p<0,05$, corregido FWE). La barra de color muestra los valores T asociados. D: derecha; I: izquierda 


\section{Discusión y conclusiones}

Los resultados de nuestro estudio muestran una reducción de sustancia gris en el córtex prefrontal lateral, incluyendo áreas inferiores, medias y superiores, el lóbulo temporal superior y la ínsula en participantes con una alta SR. Además, a nivel subcortical también observamos una reducción en el volumen del estriado ventral. Estos resultados corroboran los datos de investigaciones previas, donde se han estudiado las diferencias anatómicas asociadas a la reactividad del sistema de recompensa (Barrós-Loscertales y cols., 2006), a la impulsividad (Muhlert y Lawrence, 2015) y también al abuso de sustancias (Barrós-Loscertales y cols., 2011; Moreno-López y cols., 2012). Así pues, los resultados de nuestro estudio van en la línea de investigaciones previas, donde se observan diferencias estructurales en áreas como el córtex prefrontal, relacionado con las funciones ejecutivas y el control inhibitorio; el lóbulo temporal superior, que está implicado en la empatía y en procesos de cognición social; y, por úlitmo, la ínsula, que se asocia con el procesamiento emocional, la interocepción y la toma decisiones (Kennis y cols., 2013; Noël, Brevers y Bechara, 2013).

Los resultados derivados de los análisis de VBM se entienden como un reflejo del volumen de las estructuras biológicas subyacentes, que en el caso de la sustancia gris está formada por cuerpos celulares neuronales, neuropilo, células gliales y capilares (Yokum, Ng y Stice, 2012). En este sentido, diferencias en el volumen de sustancia gris pueden asociarse con la funcionalidad del área específica (Makris y cols., 2004). En este estudio hemos observado un decremento de volumen en el córtex prefrontal, el lóbulo temporal superior y la ínsula en participantes con un SAC sobreactivado. Estas estructuras se han visto implicadas en procesos de control cognitivo e inhibición conductual (Dixon y Christoff, 2014; Noël y cols., 2013). En esta línea, estudios de RMf han encontrado activaciones en áreas frontales inferiores e insulares en tareas de inhibición de respuesta, como por ejemplo tareas "go/no-go», tareas de señal de stop (del inglés, stop-signal task) o tareas Stroop (véase el meta-análisis de Cieslik, Mueller, Eickhoff, Langner y Eickhoff, 2015). En base a esto, la reducción de sustancia gris en las áreas indicadas podría relacionarse con una menor inhibición conductual y, por tanto, con una mayor impulsividad (Muhlert y Lawrence, 2015).

Por otra parte, las diferencias anatómicas observadas en el estriado ponen de manifiesto la implicación de esta región en la actividad del SAC (véase Kennis y cols., 2013 para revisión). Estas diferencias también se han visto en estudios funcionales, donde se ha observado una mayor activación de esta región durante el procesamiento de recompensas en personas con un una alta SR (Avila y cols., 2012; Costumero y cols., 2013). En línea con estos resultados, en un estudio reciente Caprioli y cols. (2014) observaron una reducción en el volumen de sustancia gris en el estriado ventral izquierdo en un modelo animal de impulsividad. Estos mismos autores también encontraron que esta reducción estaba acompañada por un decremento en los niveles de expresión génica de ácido gamma-aminobutírico (GABA) en esta región, lo cual asociaron con una menor proyección de neuronas gabaérgicas inhibitorias hacia áreas tálamo-corticales motoras y, por tanto, con una mayor impulsividad (Caprioli y cols., 2014; Muhlert y Lawrence, 2015). Teniendo en cuenta estos estudios previos, la reducción en el volumen de sustancia gris en áreas estriatales observadas en participantes con una alta SR podría relacionarse con una mayor respuesta hacia estímulos reforzantes (Barrós-Loscertales y cols., 2006). De esta forma, los individuos con una mayor SR podrían generar mayores respuestas de aproximación ante estímulos apetitivos, más conductas impulsivas y, en casos extremos, podrían desarrollar trastornos del control de impulsos, como TDAH (Castellanos, 2002; Tremols y cols., 2008), obesidad (Shott y cols., 2014) o abuso de sustancias (BarrósLoscertales y cols., 2011; Moreno-López y cols., 2012). 
Por último, también es importante mencionar que nuestro estudio tiene limitaciones. Una de ellas está implícita en los procedimientos de VBM, puesto que, aunque los algoritmos en SPM se consideran robustos, este software no fue inicialmente diseñado para evaluar anormalidades estructurales, y un registro imperfecto podría llevar a imprecisiones (Bookstein, 2001). No obstante, tratamos de solucionar este problema usando una versión optimizada de VBM, creando una plantilla personalizada para lograr una normalización espacial más precisa. Otra de las limitaciones está relacionada con la muestra del estudio, puesto que solo se seleccionaron hombres. Por ello, consideramos que son necesarias más investigaciones en este campo, a fin de poder comprender mejor las bases anatómicas asociadas al SAC tanto en hombres como en mujeres.

\section{Referencias bibliográficas}

Ashburner, J. y Friston, K. J. (2005). Unified segmentation. Neurolmage, 26, 839-851.

Avila, C., Garbin, G., Sanjuán, A., Forn, C., Barrós-Loscertales, A., Bustamante, J. C. y cols. (2012). Frontostriatal response to set switching is moderated by reward sensitivity. Social Cognitive and Affective Neuroscience, 7, 423-430.

Balconi, M., Finocchiaro, R. y Campanella, S. (2014). Reward sensitivity, decisional bias, and metacognitive deficits in cocaine drug addiction. Journal of Addiction Medicine, 8, 399-406.

Barrós-Loscertales, A., Garavan, H., Bustamante, J. C., Ventura-Campos, N., Llopis, J. J., Belloch, V. y cols. (2011). Reduced striatal volume in cocaine-dependent patients. Neurolmage, 56, 1021-1026.

Barrós-Loscertales, A., Meseguer, V., Sanjuán, A., Belloch, V., Parcet, M. A., Torrubia, R. y cols. (2006). Striatum gray matter reduction in males with an overactive behavioral activation system. The European Journal of Neuroscience, 24, 2071-2074.

Barrós-Loscertales, A., Ventura-Campos, N., Sanjuán-Tomás, A., Belloch, V., Parcet, M.-A. y Avila, C. (2010). Behavioral activation system modulation on brain activation during appetitive and aversive stimulus processing. Social Cognitive and Affective Neuroscience, $5,18-28$.

Bookstein, F. L. (2001). "Voxel-based morphometry" should not be used with imperfectly registered images. Neurolmage, 14, 1454-1462.

Caprioli, D., Sawiak, S. J., Merlo, E., Theobald, D. E. H., Spoelder, M., Jupp, B. y cols. (2014). Gamma aminobutyric acidergic and neuronal structural markers in the nucleus accumbens core underlie trait-like impulsive behavior. Biological Psychiatry, 75, 115-123.

Carver, C. S. y White, T. L. (1994). Behavioral inhibition, behavioral activation, and affective responses to impending reward and punishment: The BIS/BAS Scales. Journal of Personality and Social Psychology, 67, 319-333.

Castellanos, F. X. (2002). Developmental Trajectories of Brain Volume Abnormalities in Children and Adolescents With Attention-Deficit/Hyperactivity Disorder. JAMA, 288, 1740.

Cieslik, E. C., Mueller, V. I., Eickhoff, C. R., Langner, R. y Eickhoff, S. B. (2015). Three key regions for supervisory attentional control: Evidence from neuroimaging meta-analyses. Neuroscience and Biobehavioral Reviews, 48, 22-34.

Cohen, M. X. (2007). Individual differences and the neural representations of reward expectation and reward prediction error. Social Cognitive and Affective Neuroscience, 2, 20-30.

Corr, P. J. (2004). Reinforcement sensitivity theory and personality. Neuroscience and Biobehavioral Reviews, 28, 317-332. 
Costumero, V., Barrós-Loscertales, A., Bustamante, J. C., Ventura-Campos, N., Fuentes, P., Rosell-Negre, P. y cols. (2013). Reward sensitivity is associated with brain activity during erotic stimulus processing. Plos One, 8, e66940.

Depue, R. A. y Collins, P. F. (1999). Neurobiology of the structure of personality: dopamine, facilitation of incentive motivation, and extraversion. The Behavioral and Brain Sciences, 22, 491-569.

Dixon, M. L. y Christoff, K. (2014). The lateral prefrontal cortex and complex value-based learning and decision making. Neuroscience and Biobehavioral Reviews, 45, 9-18.

Franken, I. H. A., Muris, P. y Georgieva, I. (2006). Gray's model of personality and addiction. Addictive Behaviors, 31, 399-403.

Good, C. D., Johnsrude, I. S., Ashburner, J., Henson, R. N., Friston, K. J. y Frackowiak, R. S. (2001). A voxel-based morphometric study of ageing in 465 normal adult human brains. Neurolmage, 14, 21-36.

Gray, J. A. (1982). The neuropsychology of anxiety: An inquiry into the functions of the septalhippocampal system. Oxford: Oxford University Press.

Gray, J. A. y McNaughton, N. J. (2000). The neuropsychology of anxiety: An inquiry into the functions of the septo-hippocampal system. Oxford: Oxford University Press.

Haber, S. N. y Knutson, B. (2010). The reward circuit: linking primate anatomy and human imaging. Neuropsychopharmacology, 35, 4-26.

Kennis, M., Rademaker, A. R. y Geuze, E. (2013). Neural correlates of personality: an integrative review. Neuroscience and Biobehavioral Reviews, 37, 73-95.

Kimbrel, N. A., Nelson-Gray, R. O. y Mitchell, J. T. (2007). Reinforcement sensitivity and maternal style as predictors of psychopathology. Personality and Individual Differences, 42, 1139-1149.

Liu, X., Hairston, J., Schrier, M. y Fan, J. (2011). Common and distinct networks underlying reward valence and processing stages: a meta-analysis of functional neuroimaging studies. Neuroscience and Biobehavioral Reviews, 35, 1219-1236.

Makris, N., Gasic, G. P., Seidman, L. J., Goldstein, J. M., Gastfriend, D. R., Elman, I. y cols. (2004). Decreased absolute amygdala volume in cocaine addicts. Neuron, 44, 729-740.

Maldjian, J. A., Laurienti, P. J., Kraft, R. A. y Burdette, J. H. (2003). An automated method for neuroanatomic and cytoarchitectonic atlas-based interrogation of fMRI data sets. Neurolmage, 19, 1233-1239.

Mitchell, J. T. y Nelson-Gray, R. O. (2006). Attention-Deficit/Hyperactivity Disorder symptoms in adults: Relationship to Gray's Behavioral Approach System. Personality and Individual Differences, 40, 749-760.

Moreno-López, L., Catena, A., Fernández-Serrano, M. J., Delgado-Rico, E., Stamatakis, E. A., Pérez-García, M. y cols. (2012). Trait impulsivity and prefrontal gray matter reductions in cocaine dependent individuals. Drug and Alcohol Dependence, 125, 208-214.

Muhlert, N. y Lawrence, A. D. (2015). Brain structure correlates of emotion-based rash impulsivity. Neurolmage, 115, 138-146.

Noël, X., Brevers, D. y Bechara, A. (2013). A neurocognitive approach to understanding the neurobiology of addiction. Current Opinion in Neurobiology, 23, 632-638.

Pickering, A. D. y Gray, J. A. (2001). Dopamine, appetitive reinforcement, and the neuropsychology of human learning: an individual differences approach. En A. Eliasz y A. Angleitner (eds.), Advances in Individual Differences Research (pp. 113-149). Lengerich, Germany: PABST Science Publishers.

Pinto-Meza, A., Caseras, X., Soler, J., Puigdemont, D., Pérez, V. y Torrubia, R. (2006). Behavioural Inhibition and Behavioural Activation Systems in current and recovered major depression participants. Personality and Individual Differences, 40, 215-226. 
Robinson, T. E. y Berridge, K. C. (2008). Review. The incentive sensitization theory of addiction: some current issues. Philosophical Transactions of the Royal Society of London. Series B, Biological Sciences, 363, 3137-3146.

Schultz, W. (2000). Reward Processing in Primate Orbitofrontal Cortex and Basal Ganglia. Cerebral Cortex, 10, 272-283.

Sescousse, G., Caldú, X., Segura, B. y Dreher, J.-C. (2013). Processing of primary and secondary rewards: a quantitative meta-analysis and review of human functional neuroimaging studies. Neuroscience and Biobehavioral Reviews, 37, 681-696.

Shott, M. E., Cornier, M.-A., Mittal, V. A., Pryor, T. L., Orr, J. M., Brown, M. S. y cols. (2014). Orbitofrontal cortex volume and brain reward response in obesity. International Journal of Obesity (2005), 39, 214-221.

Torrubia, R., Ávila, C., Moltó, J. y Caseras, X. (2001). The Sensitivity to Punishment and Sensitivity to Reward Questionnaire (SPSRQ) as a measure of Gray's anxiety and impulsivity dimensions. Personality and Individual Differences, 31(6), 837-862.

Tremols, V., Bielsa, A., Soliva, J.-C., Raheb, C., Carmona, S., Tomas, J. y cols. (2008). Differential abnormalities of the head and body of the caudate nucleus in attention deficit-hyperactivity disorder. Psychiatry Research, 163, 270-278.

Vezina, P. (2004). Sensitization of midbrain dopamine neuron reactivity and the self-administration of psychomotor stimulant drugs. Neuroscience and Biobehavioral Reviews, 27, 827-839.

Yokum, S., Ng, J. y Stice, E. (2012). Relation of regional gray and white matter volumes to current BMI and future increases in BMI: a prospective MRI study. International Journal of Obesity (2005), 36, 656-664. 